\title{
openheart Interleukin 6 trans-signalling and the risk of future cardiovascular events in men and women
}

\author{
Yasmin Miri (D) , ${ }^{1}$ Karin Leander (D) , ${ }^{2}$ Per Eriksson, ${ }^{1}$ Bruna Gigante, ${ }^{1}$ \\ Louise Ziegler (iD ${ }^{3}$
}

\begin{abstract}
- Additional supplemental material is published online only. To view, please visit the journal online (http://dx.doi.org/10. 1136/openhrt-2021-001694).
\end{abstract}

To cite: Miri Y, Leander $\mathrm{K}$, Eriksson P, et al. Interleukin 6 trans-signalling and the risk of future cardiovascular events in men and women. Open Heart 2021;8:e001694. doi:10.1136/ openhrt-2021-001694

Received 16 April 2021 Accepted 23 September 2021

\section{Check for updates}

(c) Author(s) (or their employer(s)) 2022. Re-use permitted under CC BY. Published by BMJ.

${ }^{1}$ Division of Cardiovascular Medicine, Karolinska Institutet, Stockholm, Sweden ${ }^{2}$ Institute of Environmental Medicine, Karolinska Institutet, Stockholm, Sweden

${ }^{3}$ Department of Clinical Sciences Danderyd Hospital, Karolinska Institutet, Stockholm, Sweden

Correspondence to Dr Louise Ziegler; louise. dencker-ziegler@sll.se

\section{ABSTRACT}

Objective Pro-inflammatory interleukin 6 (IL6) trans-signalling is associated with increased risk of cardiovascular events (CVEs). Whether this association exists for both sexes is, however, uncertain. Hence, we analysed the risk of CVE associated with IL6 transsignalling in men and women and investigated if potential interaction between IL6 trans-signalling and sex affects the risk.

Methods In a prospective cohort of 60-year-old men and women without cardiovascular disease $(\mathrm{men}=2039$, women=2193), subjects were followed for 20 years. To assess the IL6 trans-signalling activity, the proportion between the active binary and inactive ternary IL6 complexes, the binary/ternary ratio $(\mathrm{B} / \mathrm{T}$ ratio), was estimated. CVE (myocardial infarction, angina pectoris and ischaemic stroke, $n=629$ ) risk was analysed with Cox regression, presented as $\mathrm{HRs}$ with $95 \% \mathrm{Cls}$. B/T ratio was dichotomised, with levels >median representing IL6 transsignalling. Interaction was analysed on the additive scale and expressed as the synergy index (S). Analyses were adjusted for cardiovascular risk factors.

Results $\mathrm{B} / \mathrm{T}$ ratio $>$ median was associated with increased CVE risk in men (HR 1.63; 95\% Cl 1.32 to 2.01), but not in women (HR 1.21; $95 \% \mathrm{Cl} 0.93$ to 1.57). There was a significant synergistic interaction $(\mathrm{S}=1.98 ; 95 \% \mathrm{Cl} 1.15$ to 3.42 ) between the $\mathrm{B} / \mathrm{T}$ ratio and male sex, the combination increasing the risk by $88 \%$.

Conclusions Our results suggest differential susceptibility to inflammation mediated by IL6 trans-signalling and subsequent CVE in men and women. The $\mathrm{B} / \mathrm{T}$ ratio could be considered as a novel biomarker for cardiovascular risk in men, but not in women.

\section{INTRODUCTION}

Sex differences in cardiovascular disease (CVD) pathophysiology, clinical presentation and prognosis are well known; for example, women display a higher prevalence of nonobstructive coronary heart disease such as stress-related cardiomyopathy, vasospasm and spontaneous coronary artery dissection. ${ }^{12}$

Inflammation plays a pivotal role in the pathophysiology of atherosclerosis. Recently, the CANTOS study showed that inhibiting interleukin (IL) $1 \beta$ in the inflammatory

\section{Key questions}

What is already known about this subject?

- Atherosclerosis is an inflammatory disease and pro-inflammatory interleukin 6 trans-signalling is associated with an increased risk of cardiovascular events. It is well known that the risk of cardiovascular disease differs between men and women. Yet, it is uncertain whether IL6 trans-signalling mediates the same effect in both sexes.

What does this study add?

- This prospective cohort study provides insight into the differential association between cardiovascular risk and inflammation driven by IL6 trans-signalling in men and women.

How might this impact on clinical practice?

- We found that men had an association between biomarkers of IL6 trans-signalling and an increased risk of CVE. The association could not be found in women. With the finding that men experience detrimental effects from IL6 trans-signalling as opposed to women, we conclude that men could possibly benefit from preventive anti-inflammatory treatment targeting IL6 trans-signalling and the IL6 transsignalling biomarkers could thus be used to identify men suitable for treatment.

IL1 $\beta-I L 6-C$ reactive protein (CRP) pathway results in beneficial effects in preventing future cardiovascular events (CVEs) in patients with inflammatory activity. ${ }^{3}$ However, in cardiovascular clinical studies women are underrepresented.

Downstream from IL1 $\beta$ is IL6, a cytokine known to mediate disparate processes depending on which of its two signalling pathways is active, classic signalling or transsignalling. In classic IL6 signalling, IL6 binds to the membrane-bound IL6 receptor (IL6R), expressed on hepatocytes, leucocytes and $\mathrm{T}$ cells. The signal is transduced by the binding to the ubiquitously expressed membranebound glycoprotein 130 (gp130). ${ }^{4}$ Classical IL6 signalling induces the acute-phase 
reaction with the production of CRP and mediates tissue homeostatic and anti-inflammatory effects. ${ }^{4}$ In IL6 transsignalling, on the other hand, IL6 binds to a soluble IL6R isoform (sIL6R) forming the circulating IL6:sIL6R (binary) complex hence enabling a systemic scope of impact. ${ }^{5}$ IL6 trans-signalling possesses detrimental proinflammatory effects and is regulated by the soluble gp130 (sgp130) binding the binary complex and forming the inactive IL6:sIL6R:sgp130 (ternary) complex. ${ }^{5}$

Our group recently explored the risk of future CVE associated with pro-inflammatory IL6 trans-signalling using a novel biomarker consisting of a ratio between the active binary IL6:sIL6R complex and the inactive ternary IL6:sIL6R:sgp130 complex, the binary/ternary complex ratio $\left(\mathrm{B} / \mathrm{T}\right.$ ratio).$^{6}$ We found that a $\mathrm{B} / \mathrm{T}$ ratio $>$ the median, mirroring a relative excess of the active binary IL6 complex, was associated with an increased risk of first-time CVE. ${ }^{6}$

In a study of men and women without established CVD, women had higher pro-inflammatory markers compared with men. ${ }^{7}$ In light of this study and clinical studies demonstrating differences in CVD risk in men and women, we hypothesised that the impact of IL6 transsignalling on CVD risk differs between men and women.

The aim of the present study was to analyse the risk of first-time CVE associated with IL6 trans-signalling in men and women, respectively. In addition, this study aims to investigate if potential differences in risk associated with $\mathrm{B} / \mathrm{T}$ ratio are dependent on the interaction between biological sex and IL6 trans-signalling.

\section{MATERIALS AND METHOD}

The study was designed and conducted as a prospective cohort study in accordance with the Declaration of Helsinki. All study participants were thoroughly informed about the study before giving their informed consent and entering the study.

\section{Study population}

From the Swedish population register, every third man and woman turning 60 and living in the Stockholm County between 1 July 1997 and 30 June 1998 were randomly selected and invited to participate in a cardiovascular health screening study. A total of 4232 subjects (2039 men and 2193 women) replied positively (78\%) and were included. A questionnaire with information regarding lifestyle, current and previous diseases, and medication was filled out by the participants. All subjects underwent a thorough physical examination, an ECG and blood sampling. The cohort is described in detail elsewhere. $^{6}$

\section{Biochemical analyses}

Fasting blood samples were collected at baseline and stored in $-80^{\circ} \mathrm{C}$ in a biobank. From the collected samples, serum levels of IL6 and sIL6R were analysed with Mesoscale Discovery Systems Cytokine Assay (Gaithersburg, MD, USA) and sgp130 with an assay development kit
(\#DY228) from R\&D Systems (R\&D Systems, Minneapolis, MN, USA). All experiments were performed according to the manufacturer's protocol. Descriptions of the experimental procedures have been previously published. ${ }^{6}$ Concentrations of IL6 were expressed in picograms per millilitre $(\mathrm{pg} / \mathrm{mL})$ and sIL6R and sgp130 in nanograms per millilitre $(\mathrm{ng} / \mathrm{mL})$. Owing to the fact that IL6, sIL6R and sgp130 interact on a molar level, the molar concentrations (moles per litre) of the binary (IL6:sIL6R) and the ternary (IL6:sIL6R:sgp130) complex, both expressed in nanomoles per litre, were estimated. Formulas presented by Garbers and Müller-Newen were used for this purpose and the calculations have been described in detail in prior publications. ${ }^{689}$

\section{Outcome}

The personal identification numbers of the subjects were linked to the national Swedish registers, the Hospital Discharge Register and the National Cause of Death Register, to extract diagnoses. Main diagnoses were recorded until 31 December 2017. The outcome was firsttime fatal or non-fatal CVE with the following diagnosis codes from the International Classification of Diseases 10th revision: myocardial infarction (MI) (I21), coronary heart disease (I20 and I25), sudden cardiac death (I46) and ischaemic stroke (I63). After excluding subjects with incomplete questionnaires $(n=122)$, lacking serum samples $(\mathrm{n}=96)$ and those with prevalent CVD $(\mathrm{n}=369$, men $=225$, women=144), there were 654 incident CVE cases. Additional 26 subjects were restricted from analyses due to inaccurately having been classified as cases, leaving 3619 subjects with 629 cases of fatal and non-fatal CVE (MI $n=221$, hospitalised angina pectoris $n=202$, cardiac arrest $n=3$ and ischaemic stroke $n=203$ ) in the final analysis. For a detailed description of the included/ excluded study participants, please see online supplemental figure 1 .

\section{Statistical analysis}

Continuous variables are presented as median and IQR. Binary variables are presented as percentages.

The relative risk of first-time CVE associated with IL6, sIL6R, sgp130 and the B/T ratio was estimated using Cox proportional hazards model and expressed as HR with 95\% CIs. The significance level was set at 5\%. All analyses were performed in men and women separately.

In initial analyses, the association between each component of the binary (IL6:sIL6R) and ternary (IL6:sIL6R:sgp130) complex and the outcome was analysed. Each component was analysed both as a continuous variable and categorised into quartiles. The quartile boundaries for the individual components in each sex can be found in online supplemental table 1 .

The risk of CVE associated with the $\mathrm{B} / \mathrm{T}$ ratio was first analysed with the $\mathrm{B} / \mathrm{T}$ ratio as a continuous variable with 0.1 unit increase due to the narrow range of the variable (1.29-2.29) and in additional analyses the $\mathrm{B} / \mathrm{T}$ ratio was categorised into quartiles. Based on the results from these 
analyses, the $\mathrm{B} / \mathrm{T}$ ratio was dichotomised at the median for men (1.59) and women (1.58), respectively, and the association between the $\mathrm{B} / \mathrm{T}$ ratio $>$ median, mirroring a relative excess of the active binary complex in relation to the inactive ternary complex and CVE risk, was analysed with the reference group being $\mathrm{B} / \mathrm{T}$ ratio $\leq$ median.

The analysis of potential interaction between the two dichotomised exposures, sex (female vs male) and IL6 trans-signalling $(\mathrm{B} / \mathrm{T}$ ratio $\leq \mathrm{vs}>$ median $)$, was made on the additive scale. The four groups of exposures were as follows: women with $\mathrm{B} / \mathrm{T}$ ratio $\leq$ median (reference group); women with $\mathrm{B} / \mathrm{T}$ ratio $>$ median; men with $\mathrm{B} / \mathrm{T}$ ratio $\leq$ median; men with $\mathrm{B} / \mathrm{T}$ ratio $>$ median. To assess interaction on an additive scale, the synergy index $(\mathrm{S})$ is presented with $S=1$ indicating an absence of interaction, $\mathrm{S}>1$ suggesting a synergistic effect and $\mathrm{S}<1$ an antagonistic effect between the exposures. $\mathrm{S}$ is presented with 95\% CI and $\mathrm{p}$ value with a significance level set at $5 \%$. An extended interaction analysis, including the relative excess risk due to interaction (RERI) and attributable portion (AP), is presented in online supplemental material.

All analyses are presented in univariate and multivariate models. In the latter, hypertension was defined as blood pressure $>140 / 90 \mathrm{mmHg}$ and/or medication for hypertension and/or self-reported in the questionnaire, diabetes mellitus as fasting glucose $>7.0 \mathrm{mmol} / \mathrm{L}$ and/ or treatment for diabetes mellitus and/or self-reported, hyperlipidaemia as fasting total serum cholesterol $>5.0$ $\mathrm{mmol} / \mathrm{L}$ and/or treatment for hyperlipidaemia and/ or self-reported. In addition, the use of menopausal hormone therapy (MHT) by female post-menopausal participants was self-reported in the questionnaire.

A complete-case analysis was performed to analyse the presence of potential effects of missing data on the results. No indication of such influence was found (data not shown).

All analyses were performed using StataCorp (Stata Statistical Software: Release 14. College Station, TX: StataCorp LP).

\section{Patient and public involvement}

This study is a population-based cohort study where every third 60-year-old man and woman living in Stockholm County was invited to participate in a cardiovascular health screening study.

\section{RESULTS}

The clinical and biochemical baseline characteristics of the study population are shown in table 1 . Men were overrepresented in the group of participants who experienced a CVE during follow-up. Men also had a higher prevalence of hyperlipidaemia and diabetes mellitus. IL6, sIL6R and sgp130 were all measured in higher concentrations in men compared with women. The $\mathrm{B} / \mathrm{T}$ ratio level was, however, similar between the sexes.

\section{IL6, sIL6R, sgp130 and the risk of future CVE}

There was a linear association between IL6 and sIL6R, respectively, and the risk of future CVE in men as seen in online supplemental table 2. A pattern of linear association with the outcome could be discerned for IL6 and sIL6R also in women although without statistical certainty (online supplemental table 3). The association between sgp130 and the outcome was not significant in either sex although the association analysis suggests a non-linear pattern (online supplemental tables 2 and 3).

\section{$B / T$ ratio and the risk of future CVE}

In men, each 0.1 unit increase of the $\mathrm{B} / \mathrm{T}$ ratio was associated with an increased risk (adjusted HR 1.58; 95\% CI 1.27 to 1.97 ) as was each $\mathrm{B} / \mathrm{T}$ ratio quartile increase (adjusted HR 1.20; 95\% CI 1.10 to 1.31 ), please see online supplemental table 4 . When analysing the risk associated with each quartile of the $\mathrm{B} / \mathrm{T}$ ratio, with the lowest quartile as the reference group, a significant risk increase was seen for $\mathrm{B} / \mathrm{T}$ ratio levels above the median (online supplemental table 2 ). In table 2 , the $\mathrm{B} / \mathrm{T}$ ratio was dichotomised at the median and levels $>$ median were associated with a $58 \%$ risk increase.

In women, results from the same analyses demonstrated smaller risk estimates without statistical significance, although the pattern of association was similar to that in men (table 2, online supplemental tables 3 and 4 ).

\section{Interaction between sex and IL6 trans-signalling on the risk of CVE}

To evaluate whether biological sex potentially modified the association between IL6 trans-signalling and the risk of CVE, the risk in different exposure groups was estimated. The group with lowest expected risk, female sex and $\mathrm{B} / \mathrm{T}$ ratio $\leq$ median, was used as the reference. As shown in figure 1 , when adding the exposure of $\mathrm{B} / \mathrm{T}$ ratio $>$ median to female sex there was a small and statistically insignificant risk increase. Instead, the combination of male sex and $\mathrm{B} / \mathrm{T}$ ratio $\leq$ median was associated with a significant risk increase. The highest risk was seen for the combination of male sex and $\mathrm{B} / \mathrm{T}$ ratio $>$ median and the risk estimate in this group was greater than the sum of the individual risks together, indicating an interaction between the two exposures. Synergy index $>1$ confirmed an interaction between male sex and IL6 trans-signalling on the risk of CVE.

\section{DISCUSSION}

This is the first study exploring the association of proinflammatory IL6 trans-signalling, mirrored by the $\mathrm{B} / \mathrm{T}$ ratio, with the risk of future CVE in men and women, respectively. The main finding is that IL6 trans-signalling is associated with an increased risk of CVE in men but not in women. In addition, there is an additive interaction between the biological sex and IL6 trans-signalling on the CVE risk. 
Table 1 Baseline characteristics of the study population

\begin{tabular}{|c|c|c|c|}
\hline & All & Men & Women \\
\hline Subjects, n (\%) & 3619 & $1705(47)$ & $1914(53)$ \\
\hline CVE, n (\%) & 629 & $396(63)$ & $233(37)$ \\
\hline \multicolumn{4}{|l|}{ Anthropometric data } \\
\hline Body mass index $\left(\mathrm{kg} / \mathrm{m}^{2}\right)$ & $26.2(23.8-28.9)$ & $26.5(24.3-28.9)$ & $25.9(23.8-28.9)$ \\
\hline Systolic pressure $(\mathrm{mm} \mathrm{Hg})$ & $136(122-152)$ & $141(128-155)$ & $132(118-148)$ \\
\hline Diastolic pressure $(\mathrm{mm} \mathrm{Hg})$ & $84(77-91)$ & 87 (80-94) & $81(75-88)$ \\
\hline \multicolumn{4}{|l|}{ Biochemical values (mmol/L) } \\
\hline Total cholesterol & $5.9(5.3-6.6)$ & $5.8(5.1-6.5)$ & $6.1(5.4-6.7)$ \\
\hline LDL & $3.8(3.2-4.5)$ & $3.8(3.2-4.4)$ & $3.8(3.2-4.5)$ \\
\hline $\mathrm{HDL}$ & $1.5(1.2-1.7)$ & $1.3(1.1-1.5)$ & $1.6(1.4-1.9)$ \\
\hline Fasting glucose & $5.2(4.8-5.7)$ & $5.3(5.0-5.8)$ & $5.1(4.7-5.5)$ \\
\hline \multicolumn{4}{|l|}{ Cardiovascular risk factors, \% } \\
\hline Hypertension & 15.8 & 15.8 & 15.7 \\
\hline Hyperlipidaemia & 3.5 & 4.2 & 2.9 \\
\hline Diabetes mellitus & 6.1 & 8.5 & 4.1 \\
\hline Current smoking & 21 & 20 & 22 \\
\hline \multicolumn{4}{|l|}{ Ongoing medical treatment, \% } \\
\hline Menopausal hormone therapy & NA & NA & 9.0 \\
\hline \multicolumn{4}{|l|}{ IL6 system biomarkers } \\
\hline IL6 (pg/mL) & $0.59(0.42-0.89)$ & $0.62(0.44-0.90)$ & $0.56(0.39-0.88)$ \\
\hline slL6R (ng/mL) & $33.4(27.1-41.5)$ & $35.8(29.0-44.4)$ & $31.4(25.7-38.4)$ \\
\hline sgp130 (ng/mL) & $383(320-451)$ & $396(339-459)$ & 368 (306-443) \\
\hline \multicolumn{4}{|l|}{ Binary/ternary complex ratio } \\
\hline $\mathrm{B} / \mathrm{T}$ ratio & $1.59(1.55-1.62)$ & $1.59(1.56-1.62)$ & $1.58(1.54-1.61)$ \\
\hline
\end{tabular}

Continuous variables are presented as median (IQR) and categorical variables are presented in percentages. CVE $n$, $(\%)=$ number of CVEs and the proportion of CVEs in the cohort and each subgroup. Missing values (men/women): systolic and diastolic blood pressure $n=3(2 / 1), \operatorname{LDL} n=45$ (34/11), current smoking $n=44$ (20/24).

CVE, cardiovascular event; HDL, high-density lipoprotein; LDL, low-density lipoprotein; NA, not applicable.

The IL6 pathway is involved in atherosclerosis-related CVD with elevated IL6 concentrations being associated with atherosclerotic plaque formation, ${ }^{10}$ microvascular dysfunction ${ }^{11}$ and an increased risk of MI. ${ }^{12}$ High levels of circulating sIL6R are associated with an increased risk of $\mathrm{CVD}^{13}$ and adverse outcomes in patients with $\mathrm{MI},{ }^{1415}$ and is causally related to CVD in Mendelian randomisation studies. ${ }^{16}{ }^{17}$ In line with this, we show a linear association for IL6 and sIL6R with the outcome primarily in men.

Studies regarding the effects of the IL6 trans-signalling inhibitor, sgp130, are inconsistent. High concentrations of sgp130 have been associated with a decreased risk of future $\mathrm{CVE}^{1318}$ but also with a negative prognosis in coronary heart disease. ${ }^{1419}$ We previously showed a non-linear

Table 2 B/T ratio and risk of future cardiovascular events

\begin{tabular}{lllll}
\hline & Crude HR (95\% Cl) & P value & Adjusted HR (95\% Cl) & P value \\
\hline $\begin{array}{l}\text { Men } \\
\text { B/T ratio }>\text { median }\end{array}$ & $1.69(1.38$ to 2.07$)$ & $<0.001$ & $1.58(1.29$ to 1.94$)$ & $<0.001$ \\
$\begin{array}{l}\text { Women } \\
\text { B/T ratio }>\text { median }\end{array}$ & $1.34(1.03$ to 1.73$)$ & 0.03 & $1.29(1.00$ to 1.68$)$ & 0.05 \\
\hline
\end{tabular}

Risk of CVE associated with the $\mathrm{B} / \mathrm{T}$ ratio expressed as $\mathrm{HR}$ with $95 \% \mathrm{Cl}$. The reference group was $\mathrm{B} / \mathrm{T}$ ratio $\leq \mathrm{median}$. The analysis is stratified by sex and adjusted for diabetes, hypertension, hyperlipidaemia, BMl and smoking. For women, further adjustments were made for MHT.

BMI, body mass index; CVE, cardiovascular event; MHT, menopausal hormone therapy. 


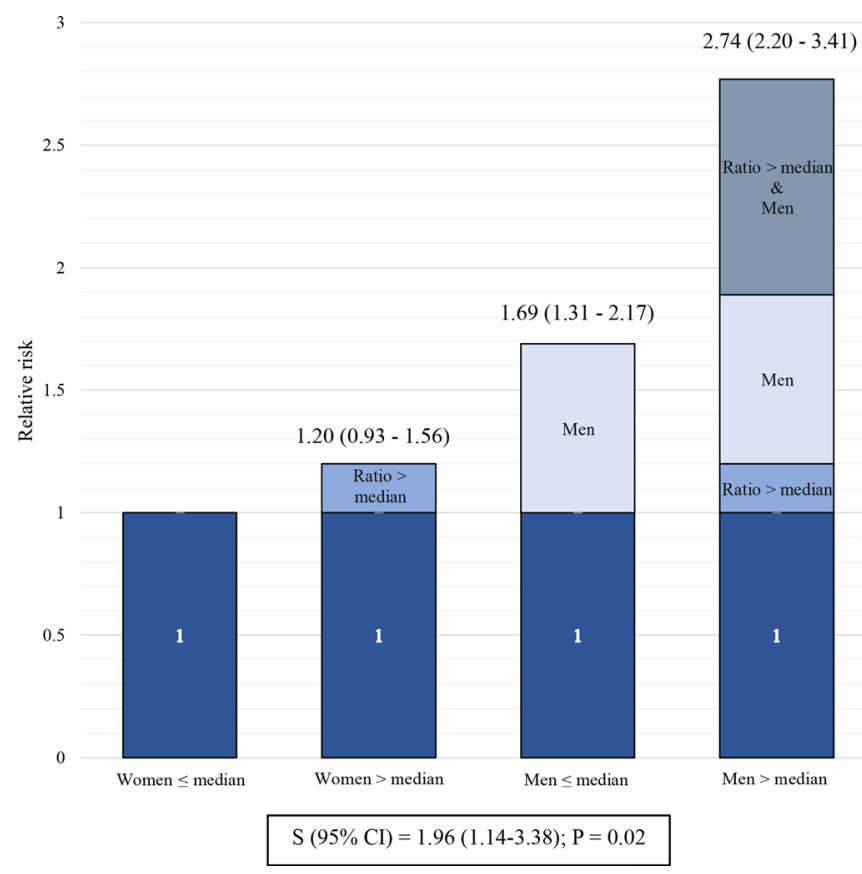

Figure 1 Interaction analysis of combined exposure of biological sex and IL6 trans-signalling. The bars graphically represent the relative risk of cardiovascular event (HR; 95\% $\mathrm{Cl}$ ) associated with different combinations of exposures of biological sex and IL6 trans-signalling. The reference group is the combination of female sex and $\mathrm{B} / \mathrm{T}$ ratio $\leq$ median (HR 1.0). The measure of additive interaction, synergy index (S), is presented with $95 \% \mathrm{Cl}$. The analysis is adjusted for diabetes, hypertension, hyperlipidaemia, body mass index and smoking.

association for sgp130 with CVE in unstratified analyses of the cohort of 60-year-olds with extremely high and low concentrations being associated with a lower CVE risk. ${ }^{6}$ Potentially elevated sgp130 levels mirror an activated IL6 trans-signalling buffer whereas low values represent a low activity of IL6 trans-signalling. In the present analysis, we found an indication of a similar non-linear association pattern in women.

Because IL6, sIL6R and sgp130 form the active binary and inactive ternary IL6 complexes on a molar level, the impact of the IL6 trans-signalling pathway cannot be evaluated with IL6 or the soluble receptors individually. We therefore constructed the $\mathrm{B} / \mathrm{T}$ ratio, a combined IL6 trans-signalling marker. In the cohort of 60-year-olds, $\mathrm{B} / \mathrm{T}$ ratio levels $>$ median, mirroring active signalling, are associated with an increased risk of a first-time $\mathrm{CVE}^{6}$ and provide prognostic information on the risk of future CVE for individuals defined as having a low-intermediate cardiovascular risk. ${ }^{20}$

In the present study, we show that $\mathrm{B} / \mathrm{T}$ ratio $>$ median is associated with an increased risk of future CVE in men but not in women.

In an Asian coronary artery disease case-control study of postmenopausal women, on the other hand, the $\mathrm{B} / \mathrm{T}$ ratio was significantly higher in cases compared with controls although an association could not be demonstrated. ${ }^{18}$ These findings together with ours indicate that the $\mathrm{B} / \mathrm{T}$ ratio may not be an appropriate predictive cardiovascular biomarker in women. Of note, the Asian study included women with acute coronary disease and thus explored the $\mathrm{B} / \mathrm{T}$ ratio as a diagnostic biomarker. Results from this study are hence not completely comparable with those in ours.

One possible explanation for the differentiating results in men and women could be sex differences related to the pathophysiology of the culprit plaque. Recent studies have shown that plaque erosion with overlying thrombosis display a lower inflammatory activity and infiltration of inflammatory cells compared with ruptured plaques. ${ }^{21}$ However, in modern studies, no difference in frequency of erosions between men and women have been demonstrated. ${ }^{21}$

The results of the interaction analysis suggest that a substantial proportion of the increased CVE risk can be attributed to the interaction between male sex and IL6 trans-signalling. One can speculate that female sex is protective of the detrimental IL6 trans-signalling effects in line with the favourable effects in systemic inflammationinduced endothelial dysfunction seen in women. ${ }^{22-24}$ The protective mechanisms are likely multifactorial, involving both genetic and hormonal factors. Several inflammatory proteins, some part of the IL1 $\beta$ pathway, are encoded on the X-chromosome. ${ }^{25}$ Compared with men, lower IL6 levels are seen in women and men with Klinefelter syndrome, both having two X-chromosomes. ${ }^{24}$ In light of this, it can be speculated that the male sex constitutes a more pro-inflammatory phenotype, with a greater susceptibility to IL6 trans-signalling and its consequences.

\section{Strengths and limitations}

This study has several limitations. Primarily, it is an observational study and thus we cannot draw any conclusions on mechanisms. Furthermore, the statistical analyses are data driven although our goal is not to establish appropriate cut-offs for the $\mathrm{B} / \mathrm{T}$ ratio but merely explore potential associations. Moreover, the inflammatory biomarkers were measured at baseline and we cannot exclude possible changes in concentrations over time. Also, we have not measured concentrations of sex hormones known to influence the development of atherosclerosis. ${ }^{26}$ In addition, some of the coronary events in the cohort could be due to non-obstructive coronary disease entailing potentially differential pathophysiology and more common in women hence possibly introducing misclassification bias. ${ }^{1}$ We do, however, not have the information on the presence of coronary plaques or plaque morphology in this study. The proportion of coronary events due to non-obstructive coronary atherosclerosis in epidemiological studies is, however, low and a large proportion of these have been shown to be caused by atherosclerosis. ${ }^{1}$ Moreover, high concentrations of IL6 have been seen in women with Takotsubo. ${ }^{27}$ In addition, since we excluded participants with prevalent CVD, the analysed group of women had lower prevalence of risk factors possibly leading to a lower incidence rate of CVE, 
thus resulting in reduced power to detect an association. Furthermore, we have not analysed the association with cardiovascular mortality but merely analysed the risk of non-fatal and fatal CVE without having enough power to perform separate analyses of the two.

The main strength of this study is that it is a large prospective population-based cohort with nearly complete 20-year follow-up. Hence, the results should be generalisable to similar populations. In addition, we have included all three components that constitute and regulate IL6 trans-signalling in one biomarker.

\section{Clinical application/future perspectives}

Lately, clinical trials have demonstrated the preventive effect of anti-inflammatory treatment by inhibiting IL1 $\beta$ and IL6 in patients with established CVD. ${ }^{32}{ }^{29}$ In the IL1 $\beta-I L 6-C R P$ pathway, only IL6 signalling has been shown to be causally associated with CVD. ${ }^{16} 17$ Moreover, recombinant sgp130 has atherosclerosis dampening properties demonstrated in experimental studies. ${ }^{30}$ Hence, targeting the pro-inflammatory IL6 trans-signalling pathway is of great interest.

Our findings suggest that men experience negative cardiovascular effects when exposed to IL6 trans-signalling and could hence benefit from anti-inflammatory treatment, for example, with recombinant sgp130. In addition, the $\mathrm{B} / \mathrm{T}$ ratio could be used as a novel biomarker to identify men with an increased cardiovascular risk and monitor treatment effects. The $\mathrm{B} / \mathrm{T}$ ratio has been seen in higher levels in women with ongoing $\mathrm{CVE},{ }^{18}$ although considering the lack of association in our study it is uncertain if women would benefit from treatment targeting IL6 trans-signalling.

\section{CONCLUSIONS}

In conclusion, IL6 trans-signalling, mirrored by the B/T ratio, is associated with an increased risk of first-time CVE in men without prevalent CVD. Accordingly, the B/T ratio could be a potential biomarker for CVE prediction and identify male individuals suitable for preventive antiinflammatory treatment. An association could, however, not be demonstrated in women. Our results suggest differential susceptibility to chronic inflammation and subsequent CVE mediated by IL6 trans-signalling in men and women.

Contributors LZ, BG and YM planned the design of the analysis. LZ and BG contributed to the data acquisition, and in addition, $\mathrm{YM}, \mathrm{KL}$ and PE performed the data analysis and interpreted the results. LZ and YM drafted the manuscript, LZ being overall responsible for the content, and all the other authors critically revised it.

Funding This work was supported by the Stockholm County Council ALF project, Strategic Research in Epidemiology at Karolinska Institutet and the Memorial Foundation of Sigurd and Elsa Golje (to BG).

\section{Competing interests None declared.}

Patient consent for publication Not applicable.

Ethics approval The study was approved by the Regional Ethics Review Board at Karolinska Institutet, Stockholm, Sweden (reference number 96-398).
Provenance and peer review Not commissioned; externally peer reviewed.

Data availability statement Data are available on reasonable request. Data are stored at Karolinska Institutet. Due to legal restrictions imposed by the Swedish Secrecy Act, requests for access individual data can be sent to BG (bruna.gigante@ ki.se).

Open access This is an open access article distributed in accordance with the Creative Commons Attribution 4.0 Unported (CC BY 4.0) license, which permits others to copy, redistribute, remix, transform and build upon this work for any purpose, provided the original work is properly cited, a link to the licence is given, and indication of whether changes were made. See: https://creativecommons.org/ licenses/by/4.0/.

ORCID iDs

Yasmin Miri http://orcid.org/0000-0002-5673-9950

Karin Leander http://orcid.org/0000-0002-1404-9222

Louise Ziegler http://orcid.org/0000-0001-6109-6761

\section{REFERENCES}

1 Mehilli J, Presbitero P. Coronary artery disease and acute coronary syndrome in women. Heart 2020;106:487-92.

2 Mehta LS, Beckie TM, DeVon HA, et al. Acute myocardial infarction in women: a scientific statement from the American Heart Association. Circulation 2016;133:916-47.

3 Ridker PM, Everett BM, Thuren T, et al. Antiinflammatory therapy with canakinumab for atherosclerotic disease. N Engl J Med 2017;377:1119-31.

4 Calabrese LH, Rose-John S. II-6 biology: implications for clinical targeting in rheumatic disease. Nat Rev Rheumatol 2014;10:720-7.

5 Schaper F, Rose-John S. Interleukin-6: biology, signaling and strategies of blockade. Cytokine Growth Factor Rev 2015;26:475-87.

6 Ziegler L, Gajulapuri A, Frumento P, et al. Interleukin 6 transsignalling and risk of future cardiovascular events. Cardiovasc Res 2019;115:213-21.

7 Lau ES, Paniagua SM, Guseh JS, et al. Sex differences in circulating biomarkers of cardiovascular disease. J Am Coll Cardiol 2019;74:1543-53.

8 Garbers C, Thaiss W, Jones GW, et al. Inhibition of classic signaling is a novel function of soluble glycoprotein 130 (sgp130), which is controlled by the ratio of interleukin 6 and soluble interleukin 6 receptor. J Biol Chem 2011;286:42959-70.

9 Muller-Newen G, Kuster A, Hemmann U. Soluble IL-6 receptor potentiates the antagonistic activity of soluble gp130 on IL-6 responses. J Immunol 1998;161:6347-55.

10 Schieffer B, Selle T, Hilfiker A, et al. Impact of interleukin-6 on plaque development and morphology in experimental atherosclerosis. Circulation 2004;110:3493-500.

11 Guo F, Dong M, Ren F, et al. Association between local interleukin-6 levels and slow flow/microvascular dysfunction. J Thromb Thrombolysis 2014;37:475-82.

12 Ridker PM, Rifai N, Stampfer MJ, et al. Plasma concentration of interleukin- 6 and the risk of future myocardial infarction among apparently healthy men. Circulation 2000;101:1767-72.

13 Moreno Velásquez I, Golabkesh Z, Källberg H, et al. Circulating levels of interleukin 6 soluble receptor and its natural antagonist, sgp130, and the risk of myocardial infarction. Atherosclerosis 2015;240:477-81.

14 Ritschel VN, Seljeflot I, Arnesen H, et al. Circulating levels of IL-6 receptor and gp130 and long-term clinical outcomes in ST-elevation myocardial infarction. J Am Heart Assoc 2016;5. doi:10.1161/ JAHA.115.003014. [Epub ahead of print: 1306 2016].

15 Groot HE, Hartman MHT, Gu YL, et al. Soluble interleukin 6 receptor levels are associated with reduced myocardial reperfusion after percutaneous coronary intervention for acute myocardial infarction. Cytokine 2015;73:207-12.

16 , Sarwar N, Butterworth AS, et al, IL6R Genetics Consortium Emerging Risk Factors Collaboration. Interleukin-6 receptor pathways in coronary heart disease: a collaborative meta-analysis of 82 studies. Lancet 2012;379:1205-13.

17 Interleukin-6 Receptor Mendelian Randomisation Analysis (IL6R MR) Consortium, Swerdlow DI, Holmes MV, et al. The interleukin-6 receptor as a target for prevention of coronary heart disease: a Mendelian randomisation analysis. Lancet 2012;379:1214-24.

18 Zhou M, Dai W, Cui Y, et al. Associations between the IL-6neutralizing sIL-6R-sgp130 buffer system and coronary artery disease in postmenopausal women. Ann Transl Med 2020;8:379.

19 Askevold ET, Nymo S, Ueland T, et al. Soluble glycoprotein 130 predicts fatal outcomes in chronic heart failure: analysis from the 
controlled rosuvastatin multinational trial in heart failure (CORONA). Circ Heart Fail 2013;6:91-8.

20 Ziegler $\mathrm{L}$, Frumento $\mathrm{P}$, Wallén $\mathrm{H}$, et al. The predictive role of interleukin 6 trans-signalling in middle-aged men and women at low-intermediate risk of cardiovascular events. Eur J Prev Cardiol 2020;27:122-9.

21 Libby P, Pasterkamp G, Crea F, et al. Reassessing the mechanisms of acute coronary syndromes. Circ Res 2019;124:150-60.

22 Rathod KS, Kapil V, Velmurugan S, et al. Accelerated resolution of inflammation underlies sex differences in inflammatory responses in humans. J Clin Invest 2017;127:169-82.

23 Young L, Cho L. Unique cardiovascular risk factors in women. Heart 2019;105:1656-60.

24 Lefèvre N, Corazza F, Valsamis J, et al. The number of X chromosomes influences inflammatory cytokine production following Toll-like receptor stimulation. Front Immunol 2019;10:1052.

25 Spolarics Z. The X-files of inflammation: cellular mosaicism of $\mathrm{X}$-linked polymorphic genes and the female advantage in the host response to injury and infection. Shock 2007;27:597-604.
26 Arnold AP, Cassis LA, Eghbali M, et al. Sex hormones and sex chromosomes cause sex differences in the development of cardiovascular diseases. Arterioscler Thromb Vasc Biol 2017;37:746-56.

27 Scally C, Abbas H, Ahearn T, et al. Myocardial and systemic inflammation in acute stress-induced (takotsubo) cardiomyopathy. Circulation 2019;139:1581-92.

28 Kleveland O, Kunszt G, Bratlie M, et al. Effect of a single dose of the interleukin- 6 receptor antagonist tocilizumab on inflammation and troponin $\mathrm{T}$ release in patients with non-ST-elevation myocardial infarction: a double-blind, randomized, placebo-controlled phase 2 trial. Eur Heart J 2016;37:2406-13.

29 Tardif J-C, Kouz S, Waters DD, et al. Efficacy and safety of low-dose colchicine after myocardial infarction. $N$ Engl J Med 2019;381:2497-505.

30 Schuett $\mathrm{H}$, Oestreich R, Waetzig GH, et al. Transsignaling of interleukin-6 crucially contributes to atherosclerosis in mice. Arterioscler Thromb Vasc Biol 2012;32:281-90. 